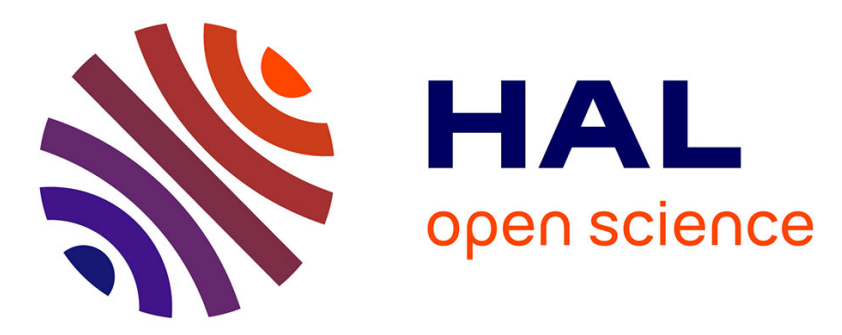

\title{
Many-body effects at the origin of structural transitions in $\mathrm{B} 2 \mathrm{O} 3$
}

\author{
Axelle Baroni, Fabien Pacaud, Mathieu Salanne, Matthieu Micoulaut, \\ Jean-Marc Delaye, Anita Zeidler, Philip Salmon, Guillaume Ferlat
}

\section{- To cite this version:}

Axelle Baroni, Fabien Pacaud, Mathieu Salanne, Matthieu Micoulaut, Jean-Marc Delaye, et al.. Manybody effects at the origin of structural transitions in B2O3. Journal of Chemical Physics, 2019. hal-02489737

\section{HAL Id: hal-02489737 \\ https://hal.science/hal-02489737}

Submitted on 24 Feb 2020

HAL is a multi-disciplinary open access archive for the deposit and dissemination of scientific research documents, whether they are published or not. The documents may come from teaching and research institutions in France or abroad, or from public or private research centers.
L'archive ouverte pluridisciplinaire HAL, est destinée au dépôt et à la diffusion de documents scientifiques de niveau recherche, publiés ou non, émanant des établissements d'enseignement et de recherche français ou étrangers, des laboratoires publics ou privés. 


\section{Many-body effects at the origin of structural transitions in $\mathrm{B}_{2} \mathrm{O}_{3}$}

Axelle Baroni, ${ }^{1,2,3}$ Fabien Pacaud, ${ }^{4}$ Mathieu Salanne, ${ }^{1}$ a) Matthieu Micoulaut, ${ }^{2}$ Jean-Marc Delaye, ${ }^{4}$ Anita

Zeidler, ${ }^{5}$ Philip S. Salmon, ${ }^{5}$ and Guillaume Ferlat ${ }^{3, \text { b) }}$

${ }^{1)}$ Sorbonne Université, CNRS, PHENIX, F-75005, Paris, France

${ }^{2)}$ Sorbonne Université, CNRS, LPTMC, F-75005, Paris, France

${ }^{3)}$ Sorbonne Université, CNRS, MNHN, IRD, IMPMC, F-75005 Paris, France

${ }^{4)}$ CEA, DEN, Laboratoire d'Etude des Matériaux et Procédés Actifs, 30207 Bagnols-sur-Cèze,

France

${ }^{5)}$ Department of Physics, University of Bath, Bath BA2 7AY, United Kingdom

(Dated: 24 February 2020)

The structural properties of glassy diboron trioxide, $\mathrm{g}-\mathrm{B}_{2} \mathrm{O}_{3}$, are investigated from ambient to high pressure conditions using two types of atomic force-field models that account for many-body effects. These models are parameterized by a dipole- and force-fitting procedure of reference data sets created via first-principles calculations on a series of configurations. The predictions of the models are tested against experimental data, where particular attention is paid to the structural transitions in $\mathrm{g}-\mathrm{B}_{2} \mathrm{O}_{3}$ that involve changes to both the short- and medium-range order. The models outperform those previously devised, where improvement originates from the incorporation of two key physical ingredients, namely (i) the polarizability of the oxide ion and (ii) the ability of an oxide ion to change both size and shape in response to its coordination environment. The results highlight the importance of many-body effects for accurately modelling this challenging system.

PACS numbers: 61.45.Fs, 62.50.-p, 64.70.kj

\section{INTRODUCTION}

Diboron trioxide, $\mathrm{B}_{2} \mathrm{O}_{3}$, is an atypical network-forming system that shows, despite its chemical simplicity, a variety of structural motifs in both its crystalline and glassy phases (see Fig. 1 and, e.g., Ref. 1 for a review). Two crystalline polymorphs have so far been reported from experiment. The structure of $\mathrm{B}_{2} \mathrm{O}_{3}-\mathrm{I}$ is based on a network of corner-sharing $\mathrm{BO}_{3}$ triangular units, ${ }^{2}$ where all of the boron atoms are three-fold coordinated $\left(\mathrm{B}_{3}\right)$, whereas the structure of $\mathrm{B}_{2} \mathrm{O}_{3}$-II is based on a network of corner-sharing $\mathrm{BO}_{4}$ tetrahedral units, ${ }^{3}$ where all of the boron atoms are four-fold coordinated $\left(\mathrm{B}_{4}\right)$. The crystallization of these polymorphs from the melt requires the application of pressure, typically above 0.4 and $2.0 \mathrm{GPa}$ for the formation of $\mathrm{B}_{2} \mathrm{O}_{3}$-I and $\mathrm{B}_{2} \mathrm{O}_{3}$-II, respectively. ${ }^{4}$ The crystal structures have mass densities of $\rho \simeq 2.56 \mathrm{~g} \mathrm{~cm}^{-3}\left(\mathrm{~B}_{2} \mathrm{O}_{3}-\mathrm{I}\right)$ and $\rho \simeq 3.11 \mathrm{~g} \mathrm{~cm}^{-3}\left(\mathrm{~B}_{2} \mathrm{O}_{3}\right.$-II). In comparison, the glassy phase, $\mathrm{g}-\mathrm{B}_{2} \mathrm{O}_{3}$, has a much smaller density $\rho \simeq 1.8 \mathrm{~g} \mathrm{~cm}^{-3}$ under ambient conditions. Here, the glass structure is dominated by boroxol rings, ${ }^{5}$ which are superstructural units of planar rings constructed from three $\mathrm{BO}_{3}$ units (Fig. 1c). These rings stabilize low-density boron oxide networks, not only in $\mathrm{g}-\mathrm{B}_{2} \mathrm{O}_{3}$ but also in several predicted crystalline phases ${ }^{6-9}$ (see Ref. 10 for a review). Thus, rich structural complexity is expected for $\mathrm{g}-\mathrm{B}_{2} \mathrm{O}_{3}$ as the pressure (or density) is changed. This expectation is confirmed by experimental investigations of the pressurised glass. A dissolution of boroxol rings is observed at low density as the rings are broken into a network of $\mathrm{BO}_{3}$ motifs. ${ }^{11-13}$ At higher density, there is a more abrupt transformation into a network of $\mathrm{BO}_{4}$ motifs. ${ }^{12-15}$ The $\mathrm{B}_{2} \mathrm{O}_{3}$

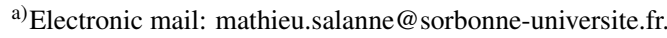

b)Electronic mail: guillaume.ferlat@sorbonne-universite.fr.
}

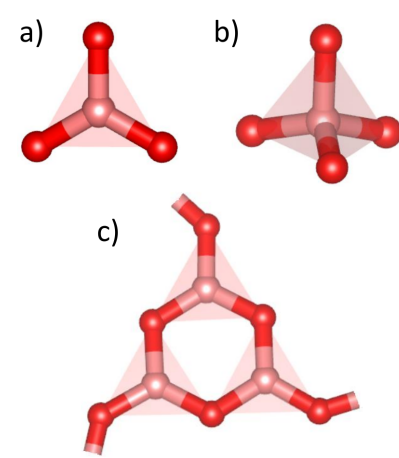

FIG. 1. Structural units found in $\mathrm{B}_{2} \mathrm{O}_{3}$ network structures, where the $\mathrm{B}$ and $\mathrm{O}$ atoms are represented by pink and red spheres, respectively. a) Three-fold coordinated boron atom $\left(\mathrm{B}_{3}\right)$; b) four-fold coordinated boron atom $\left(\mathrm{B}_{4}\right)$; c) $\mathrm{B}_{3} \mathrm{O}_{9 / 2}$ boroxol group containing a six-membered $\mathrm{B}_{3} \mathrm{O}_{3}$ boroxol ring.

system therefore provides an excellent test bed for assessing the key physical ingredients that are necessary to obtain realistic structural models of borate glasses from computer simulations.

In the past, first-principles molecular dynamics (FPMD) simulations within the density functional theory (DFT) framework have been used to investigate the structure of liquid and glassy $\mathrm{B}_{2} \mathrm{O}_{3}$ from ambient to high-pressure conditions. ${ }^{5,15-19}$ However, the affordable system sizes and time scales are limited severely by the computational cost of these simulations. Previous FPMD studies of $\mathrm{B}_{2} \mathrm{O}_{3}$ have typically been restricted to systems of about 150 atoms and trajectories of a few tens of picoseconds. Unfortunately, such short simulations prevent the structural reorganisation that is observed experimentally as the liquid is cooled or as the glass is compressed. Thus, less time demanding simulations based upon empirical force fields are highly desirable as an alternative to FPMD. 
The modelling of $\mathrm{g}-\mathrm{B}_{2} \mathrm{O}_{3}$ using empirical force fields ${ }^{6,7,13,19-40}$ was initiated four decades ago, ${ }^{20,21}$ but it has also proved challenging and remains a topical subject. ${ }^{19}$ Although the ambient glass structure has been firmly established from experiment, showing that all boron atoms are three-fold coordinated with a high proportion of them $(\sim 65$ $80 \%$ ) inside boroxol rings, ${ }^{41-43}$ all "quench-from-the-melt" models of the glass obtained from molecular dynamics (MD) simulations suffer from at least one of the following pitfalls: the presence of defects $\left(\mathrm{B}_{4}\right)$; a severe underestimation of the proportion of boroxol rings; and/or densities (or bond angles or structure factors) that are inconsistent with experiment. These failures have been attributed to imperfections of the force field and to the use of unrealistic quench rates in the simulations (see Ref. 10 for a review of the merits and weaknesses of the various models). Indeed, both aspects are entangled, and are particularly acute in the case of $\mathrm{B}_{2} \mathrm{O}_{3}$.

In respect of the force field, it should be sufficiently accurate to be able to predict both (i) the formation of boroxol rings (a many-body entity) and (ii) the ratio of $\mathrm{B}_{3}$ to $\mathrm{B}_{4}$ species present in the glass. Not surprisingly, simulations using potentials restricted to pair interactions fail to produce boroxolring containing structures, although these rings are sometimes observed in studies that employ higher-order (three- and up to four-body) interactions. ${ }^{24,26,34}$ Oftentimes, the many-body terms are incorporated via angular constraints ${ }^{19,26,30,31}$ in order to account for the directionality of the covalent bonds. Another issue is the atomic polarizability. ${ }^{13,34,40}$ It has been suggested that inter-atomic potentials should include terms accounting for the ring stabilisation energy, which is expected from delocalized $\pi$-bonding. ${ }^{41}$ However, this effect can in principle be captured implicitly through, e.g., polarizable or coordination-dependant force fields.

An assessment of the force-field quality for $\mathrm{g}-\mathrm{B}_{2} \mathrm{O}_{3}$, via simulation of the liquid to glass transformation, is obscured by incomplete equilibration. Experimentally, the fraction of boroxol rings increases rapidly as the liquid is cooled and the glass transition temperature $T_{\mathrm{g}}$ is approached, ${ }^{44,45}$ concomitant with a large increase in the viscosity. The equilibration times approach or even exceed the duration of a simulation, thus hindering to a large extent the structural changes observed experimentally. Because the formation of rings is entropically unfavorable ${ }^{46}$ it is easier numerically to explore the reverse transformation, i.e., ring dissolution, a strategy that we shall adopt in the following work.

In this paper, we will consider classical force-fields for systems of ions with full formal changes, which are carefully parameterized using the results from DFT calculations on a series of crystal and/or glass configurations. Three force fields are considered that were devised in previous studies that had different goals, aimed at investigating (i) the temperature-induced transformations in the liquid, ${ }^{40}$ (ii) the pressure-induced transformations in the glass, ${ }^{13}$ or (iii) the concentration-induced transformations in binary and ternary systems ${ }^{47}$ The force fields belong to two different families of ionic interaction models. The first is based on the polarizable ion model (PIM), where polarization effects are included but the ionic radii are fixed. The second is based on the aspher- ical ion model (AIM) where, in addition to the polarization degrees of freedom, an account of aspherical ion deformation effects is included in the short-range repulsion term. In the present work, we benchmark all three force fields within a self-consistent framework, and extend the tests that were applied previously. The validity of the models is examined by investigating the microscopic origins of the pressure-induced transformations in $\mathrm{g}-\mathrm{B}_{2} \mathrm{O}_{3}$, namely boroxol ring dissolution and the transition from $\mathrm{B}_{3}$ to $\mathrm{B}_{4}$ species. Additional tests are based on investigations of the crystal structures and nature of the ambient pressure glass.

The results highlight the ingredients that are required to capture subtle yet important many-body effects that are necessary to model accurately the structure of this challenging system. We also provide new evidence for the energetic origin of boroxol rings.

\section{METHODS}

\section{A. Interaction potentials}

At the simplest level, the pairwise additive models generally used in classical molecular dynamics (MD) simulations reproduce reasonably well the structure of many condensed phase systems. In oxides, generalized forms of the BornHuggins-Mayer potential ${ }^{48-50}$ are commonly employed: ${ }^{51-53}$

$$
V_{\mathrm{BHM}}=V_{\text {rep }}+V_{\text {Coul }}+V_{\text {disp }}
$$

where $V_{\text {rep }}=\sum_{i<j} A^{i j} \exp \left(-a^{i j} r^{i j}\right)$ is a short-range repulsion term, $V_{\text {Coul }}=\sum_{i<j} \frac{q^{i} q^{j}}{r^{i j}}$ describes the Coulomb interactions, and $q^{i}$ is the formal charge on ion $i$. The dispersion term $V_{\text {disp }}$ is usually limited to the first two leading terms of the asymptotic multipole expansion:

$$
V_{\mathrm{disp}}=-\sum_{i<j}\left[f_{6}^{i j}\left(r^{i j}\right) \frac{C_{6}^{i j}}{\left(r^{i j}\right)^{6}}+f_{8}^{i j}\left(r^{i j}\right) \frac{C_{8}^{i j}}{\left(r^{i j}\right)^{8}}\right],
$$

where $C_{6}^{i j}$ and $C_{8}^{i j}$ are the dipole-dipole and dipole-quadrupole dispersion coefficients, respectively. A correction for shortrange penetration is made using Tang-Toennies damping functions: ${ }^{54}$

$$
f_{n}^{i j}\left(r^{i j}\right)=1-\mathrm{e}^{-b_{n}^{i j} r^{i j}} \sum_{k=0}^{n} \frac{\left(b_{n}^{i j} r^{i j}\right)^{k}}{k !} .
$$

The resulting simple potentials, in which the interactions depend only on the distance between pairs of ions $r^{i j}$, are insufficient to model the structure of $\mathrm{B}_{2} \mathrm{O}_{3}$. In fact, Madden and co-workers used a series of ab initio calculations to show that in many oxide materials it is necessary to take into account many-body effects in order to reproduce the structure, as well as other properties of a system such as its vibrational 
modes. ${ }^{55-58}$ A series of models of increasing complexity were thereby devised to account for these many-body effects.

In the PIM, the response of each ion to the presence of an external field is included by attributing induced dipoles $\boldsymbol{\mu}^{i}$ to each ion $i$. This leads to an additional polarization component to the interaction potential,

$$
\begin{aligned}
V_{\mathrm{pol}}= & \sum_{i<j}\left[\frac{q^{i} \mathbf{r}^{i j} \cdot \boldsymbol{\mu}^{j}}{\left(r^{i j}\right)^{3}} g_{D}^{i j}\left(r^{i j}\right)-\frac{\boldsymbol{\mu}^{i} \cdot \mathbf{r}^{i j} q^{j}}{\left(r^{i j}\right)^{3}} g_{D}^{j i}\left(r^{i j}\right)\right. \\
& \left.+\frac{\boldsymbol{\mu}^{i} \cdot \boldsymbol{\mu}^{j}}{\left(r^{i j}\right)^{3}}-\frac{3\left(\mathbf{r}^{i j} \cdot \boldsymbol{\mu}^{i}\right)\left(\mathbf{r}^{i j} \cdot \boldsymbol{\mu}^{j}\right)}{\left(r^{i j}\right)^{5}}\right] \\
& +\sum_{i} \frac{1}{2 \alpha^{i}}\left|\boldsymbol{\mu}^{i}\right|^{2},
\end{aligned}
$$

where the first two terms describe charge-dipole interactions and the last two terms describe dipole-dipole interactions. Tang-Toennies functions ${ }^{54}$ are again used to account for shortrange effects in the charge-dipole interaction:

$$
g_{D}^{i j}\left(r^{i j}\right)=1-c_{D}^{i j} \mathrm{e}^{-b_{D}^{i j} r^{i j}} \sum_{k=0}^{n} \frac{\left(b_{D}^{i j} r^{i j}\right)^{k}}{k !} .
$$

In equation (3), the last term corresponds to the energy cost of deforming the charge density of ion $i$ of polarizability $\alpha^{i}$. The induced dipoles are treated as additional degrees of freedom, which have to be determined self-consistently at each step of the simulation. In our case, this is done by minimizing $V_{\text {pol }}$. The induced dipoles depend on the positions of all the atoms such that the polarization term has a many-body essence, despite the pairwise additive form of equation (3). The total potential in the PIM is given by

$$
V_{\mathrm{PIM}}=V_{\mathrm{BHM}}+V_{\mathrm{pol}} \text {. }
$$

The AIM is representative of a more sophisticated class of ionic interaction models, in which the repulsion term is no longer represented by a single-variable analytical function such as in the Born-Huggins-Mayer potential. Instead, additional degrees of freedom are attributed to the oxide ion, allowing it to breathe in an aspherical way, in response to its changing nearest-neighbor environment. ${ }^{59-61}$ In practice, the inter-particle distance in the pair-potential is replaced by the variable quantity

$$
\rho^{i j}=r^{i j}-\delta \sigma^{i}-\delta \sigma^{j}-\mathbb{S}_{\alpha}^{(1)}\left(v_{\alpha}^{i}-v_{\alpha}^{j}\right)-\mathbb{S}_{\alpha \beta}^{(2)}\left(\kappa_{\alpha \beta}^{i}+\kappa_{\alpha \beta}^{j}\right) .
$$

The scalar $\delta \sigma^{i}$ represents the deviation of the radius of the ion from its default value, while $v^{i}$ and $\kappa^{i}$ are sets of three and five variables that describe the dipolar and quadrupolar shape distortion, respectively. $\mathbb{S}^{(1)}$ and $\mathbb{S}^{(2)}$ are the corresponding interaction tensors:

$$
\begin{aligned}
\mathbb{S}_{\alpha}^{(1)} & =\frac{r_{\alpha}^{i j}}{r^{i j}} \\
\mathbb{S}_{\alpha \beta}^{(2)} & =\frac{3 r_{\alpha}^{i j} r_{\beta}^{i j}}{r^{i j^{2}}}-\delta_{\alpha \beta}
\end{aligned}
$$

and $\delta_{\alpha \beta}$ is the Kronecker delta.
In the case of pure $\mathrm{B}_{2} \mathrm{O}_{3}$, the resulting repulsion potential is given by:

$$
\begin{aligned}
V_{\mathrm{asph}}= & \sum_{i \in \mathrm{B}, j \in \mathrm{O}}\left[A^{+-} \exp \left(-a^{+-} \rho^{i j}\right)+B^{+-} \exp \left(-b^{+-} \rho^{i j}\right)\right] \\
& +\sum_{i \in \mathrm{O}, j \in \mathrm{O}, i<j} A^{--} \exp \left(-a^{--} \rho^{i j}\right) \\
& +\sum_{i \in \mathrm{B}, j \in \mathrm{B}, i<j} A^{++} \exp \left(-a^{++} \rho^{i j}\right) \\
& +\sum_{i \in \mathrm{O}}\left\{D\left[\exp \left(\beta \delta \sigma^{i}\right)+\exp \left(-\beta \delta \sigma^{i}\right)\right]\right. \\
& \left.+\left[\exp \left(\zeta^{2}\left|v^{i}\right|^{2}\right)-1\right]+\left[\exp \left(\eta^{2}\left|\kappa^{i}\right|^{2}\right)-1\right]\right\}
\end{aligned}
$$

where

$$
\left|\kappa^{i}\right|^{2}=\kappa_{x x}^{i{ }^{2}}+\kappa_{y y}^{i{ }^{2}}+\kappa_{z z}^{i^{2}}+2\left({\kappa_{x y}^{i}}^{2}+\kappa_{x z}^{i}{ }^{2}+\kappa_{y z}^{i{ }^{2}}\right)
$$

and the total AIM potential is given by

$$
V_{\mathrm{AIM}}=V_{\mathrm{asph}}+V_{\mathrm{Coul}}+V_{\mathrm{disp}}+V_{\mathrm{pol}} .
$$

It is worth noting that no quadrupolar terms were included in $V_{\text {pol }}$ because they did not improve the accuracy of the force field (as measured through the fitting procedure described below), and because they often lead to reduced stability of simulations at high temperatures (frequent "polarization catastrophes", i.e. numerical instabilities leading to non-physical overpolarisation, may occur ${ }^{62}$ ).

\section{B. Parameterization from DFT calculations}

The atomic or pairwise additive parameters in the PIM and AIM all have a well-defined physical origin, and a methodology has been set-up to determine them from DFT calculations alone ${ }^{63,64}$ The procedure is the following: ${ }^{65}$

1. Generate a number $N_{c}$ of typical condensed-phase configurations.

2. Perform DFT calculations on each of these configurations to:

(a) Determine the ground-state wavefunctions, which give access to the first-principles force components $\mathbf{F}_{\text {DFT }}$.

(b) Find the localized Wannier functions, from which the first-principles induced dipole components $\mu_{\mathrm{DFT}}$ are calculated.

3. Minimize the function $\chi_{D}^{2}$ with respect to the parameters of the polarization term $\left(V_{\mathrm{pol}}\right)$ where

$$
\chi_{D}^{2}=\frac{1}{N_{c}} \sum_{j=1}^{N_{c}} \frac{1}{N_{j}} \sum_{i=1}^{N_{j}} \frac{\left|\boldsymbol{\mu}_{\mathrm{DFT}}^{i}-\boldsymbol{\mu}_{\mathrm{PIM} / \mathrm{AIM}}^{i}\right|^{2}}{\left|\boldsymbol{\mu}_{\mathrm{DFT}}^{i}\right|^{2}}
$$

and $N_{j}$ is the number of atoms in configuration $j$. 

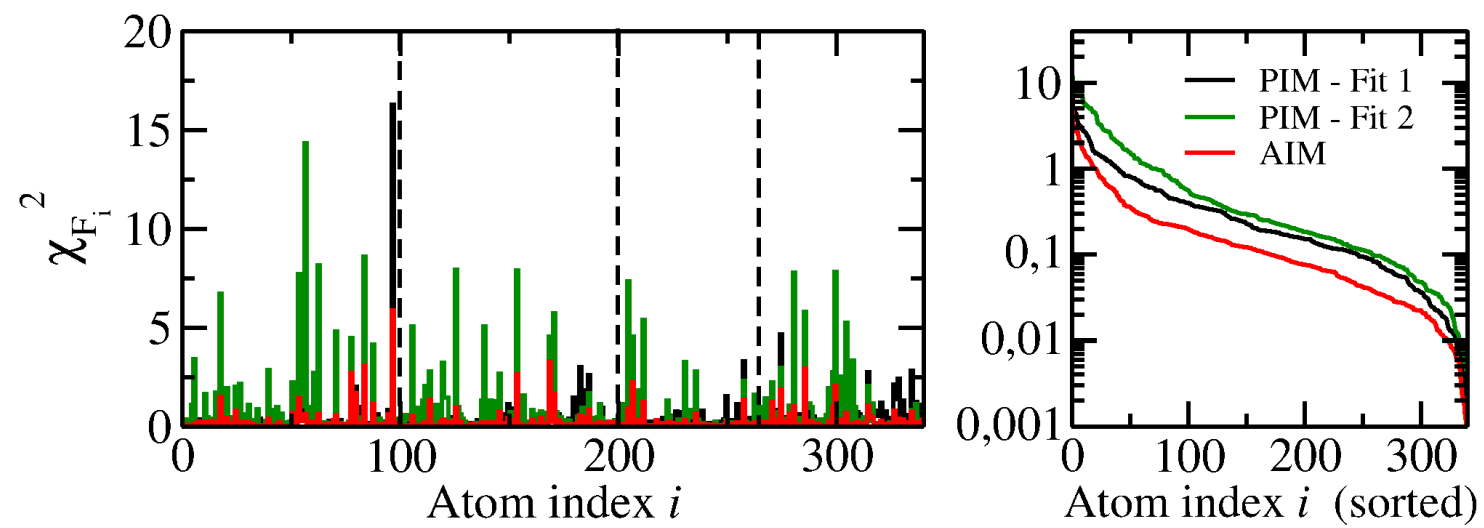

FIG. 2. Estimation of the error made by the empirical interaction potentials in determining the force on each atom relative to the force given by the reference DFT calculations. Left: Each block of data separated by vertical dashed lines corresponds to one of four different configurations, where the first two configurations correspond to glassy systems of 100 atoms, and the second two configurations correspond to orthorhombic super-cells for crystalline $\mathrm{B}_{2} \mathrm{O}_{3}$-I and $\mathrm{B}_{2} \mathrm{O}_{3}$-II. Right: The data sets sorted by decreasing value of $\chi_{F_{i}}^{2}$.

4. Minimize the function $\chi_{F}^{2}$ with respect to the parameters of the repulsion term $\left(V_{\mathrm{BHM}}\right.$ for the PIM or $V_{\text {asph }}$ for the AIM) where

$$
\chi_{F}^{2}=\frac{1}{N_{c}} \sum_{j=1}^{N_{c}} \frac{1}{N_{j}} \sum_{i=1}^{N_{j}} \frac{\left|\mathbf{F}_{\mathrm{DFT}}^{i}-\mathbf{F}_{\mathrm{PIM} / \mathrm{AIM}}^{i}\right|^{2}}{\left|\mathbf{F}_{\mathrm{DFT}}^{i}\right|^{2}}
$$

In the case of $\mathrm{B}_{2} \mathrm{O}_{3}$, the reference DFT calculations were performed using the Perdew-Burke-Ernzerhof (PBE) generalized gradient approximation (GGA) for the exchange-correlation energy. ${ }^{66}$ First, we determined the parameters of the AIM by combining dipole-fitting and force-fitting for a set of four configurations that included the crystalline polymorphs $\mathrm{B}_{2} \mathrm{O}_{3}-\mathrm{I}$ $\left(\rho=2.56 \mathrm{~g} \mathrm{~cm}^{-3}\right)$ and $\mathrm{B}_{2} \mathrm{O}_{3}$-II $\left(\rho=3.11 \mathrm{~g} \mathrm{~cm}^{-3}\right)$, and two structures of glassy $\mathrm{B}_{2} \mathrm{O}_{3}$ generated ${ }^{67}$ for the ambient conditions density $\rho=1.84 \mathrm{~g} \mathrm{~cm}^{-3}$. Note that only the $\mathrm{B}_{2} \mathrm{O}_{3}$-II configuration contained four-fold coordinated boron atoms. The $\chi_{D}^{2}$ and $\chi_{F}^{2}$ values were 0.027 and 0.263 , respectively, which indicate good overall reproduction of the DFT reference data ${ }^{65}$. (The different orders of magnitude for $\chi_{D}^{2}$ versus $\chi_{F}^{2}$ reflect mostly the fact that the dipoles have a finite average value, whereas the magnitude of the forces is centered around zero. The latter can lead to large differences for small force values.) In attempting to fit the parameters of the PIM to the same set of configurations, the difference with DFT gets much larger and some parameters take unphysical values due to large error compensations. However, by restricting the fit to the two glassy configurations alone, we could get a more well-behaved PIM potential, denoted by PIM-Fit 1 in the following. In order to gain a measure of the transferability of this potential with respect to the AIM, $\chi_{D}^{2}$ and $\chi_{F}^{2}$ were also calculated for the same set of four configurations: values of 0.031 and 0.499 were obtained, respectively.

Finally, a second PIM potential was fitted using a different and larger set of 36 configurations, chosen from seven different compositions of borosilicates, including sodium- and lithium-containing glasses. In these systems, the ratio of $\mathrm{B}_{3}$ to $\mathrm{B}_{4}$ species can be tuned by changing the alkali to boron oxide ratio. The derived model, denoted by PIM-Fit 2, has been used recently for the study of $\mathrm{Na}_{2} \mathrm{O}-\mathrm{B}_{2} \mathrm{O}_{3}-\mathrm{SiO}_{2}$ glasses. ${ }^{47}$ In its application to pure $\mathrm{B}_{2} \mathrm{O}_{3}$, the model delivers poorer performance $\left(\chi_{D}^{2}=0.030\right.$ and $\left.\chi_{F}^{2}=0.890\right)$. However, we use it in order to make a comparative assessment of the high-pressure trends.

The dispersion parameters are the only terms that are not determined from this fitting procedure. Indeed, the dispersion interaction is not accounted for well in standard GGA DFT functionals. ${ }^{68-70}$ In consequence, this term was added afterwards. Here, the O-O dispersion term was taken from the work of Jahn et al. ${ }^{61}$ but no dispersion terms were included for interactions involving the boron atom because of its small polarizability. Values for all of the parameters used in the AIM, PIM-Fit 1 and PIM-Fit 2 potentials are reported in the Appendix.

In order to analyze further the error associated with each of the models in respect to the DFT calculations, we calculated the parameter $\chi_{F_{i}}^{2}$ for each atom $i$ of each configuration. The results are given in Fig. 2, and show that the large average error of the two PIM potentials arises mostly from a few atomic positions for which the error is very large $\left(\chi_{F_{i}}^{2}>1\right.$, sometimes reaching 10 for PIM-Fit 2). This contrasts with the AIM potential, for which the errors are smaller and distributed uniformly, with only a few atomic positions for which $\chi_{F_{i}}^{2} \approx 1$. The latter correspond mostly to atoms for which the DFT force is very small, which leads to a large relative error.

\section{Simulations}

The PIM-Fit 1, PIM-Fit 2 and AIM potentials were tested using structural optimizations of the known crystals and MD simulations of the glass. Part of the high-pressure investigation using the AIM potential is reported in Ref. 13. For the glass, the initial configuration used for the MD simulations was the boroxol-ring rich (320 atom) model obtained in Ref. 5, which gives a density and structure at ambient conditions that are fully consistent with experiments. 


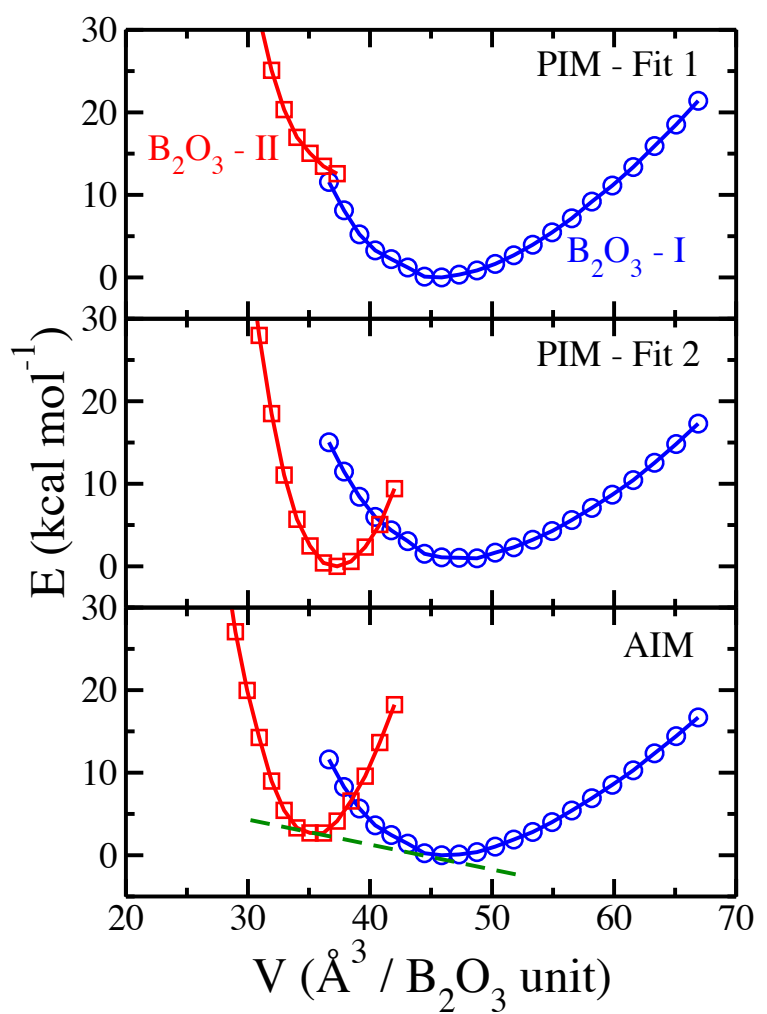

FIG. 3. Volume dependence of the energy for the crystalline structures of $\mathrm{B}_{2} \mathrm{O}_{3}-\mathrm{I}$ and $\mathrm{B}_{2} \mathrm{O}_{3}-\mathrm{II}$ at $0 \mathrm{~K}$ from the PIM-Fit 1, PIM-Fit 2 and AIM potentials. The dashed (green) line is the common tangent to the equilibrium curves.

A time step of $1 \mathrm{fs}$ was used for all the simulations, and the Coulomb and polarisation forces were calculated using the Ewald summation method. ${ }^{63,71}$ Short runs (a few tens of ps) in the NPT ensemble were performed at several target pressures. From the configurations obtained, simulations in the $N V T$ ensemble were performed for at least $1 \mathrm{~ns}$. The pressures indicated throughout this paper are the average values obtained from the $N V T$ (i.e., constant density) runs. All of the potentials used in this study reproduce very well the measured pressure dependence of the density over the entire pressure range of 0-9 GPa probed in the in-situ experiments of Brazhkin et al. ${ }^{15}$ (see Fig. 1 of Ref. 13 for the AIM case).

\section{RESULTS}

\section{A. The $B_{3}$ to $B_{4}$ transition}

Capturing the $\mathrm{B}_{3}$ to $\mathrm{B}_{4}$ transition is a challenge for empirical potentials. For example, in studies of borosilicates and related systems, where the transition is driven by compositional change, Kieu et al. ${ }^{72}$ had to adapt the force-field parameters for each composition in order to reproduce the measured speciation. In pure $\mathrm{g}-\mathrm{B}_{2} \mathrm{O}_{3}$, even coordinationdependent potentials ${ }^{39,73}$ have so far failed to reproduce the pressure threshold at which $\mathrm{B}_{4}$ species start to form ${ }^{13}$ (see below).

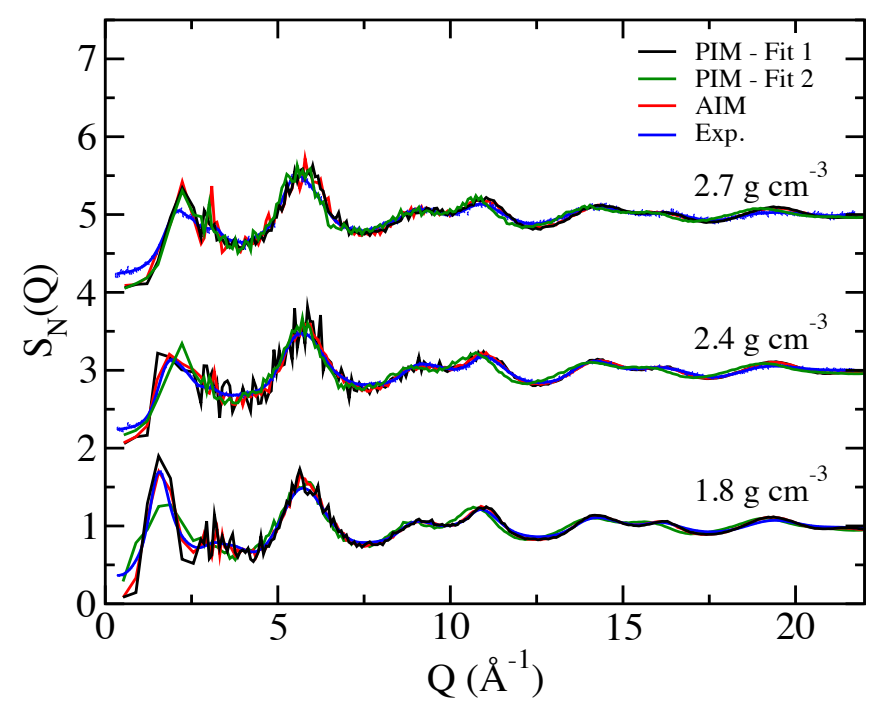

FIG. 4. Neutron total structure factors $S_{\mathrm{N}}(Q)$ for $\mathrm{g}-\mathrm{B}_{2} \mathrm{O}_{3}$ at three different densities. The experimental data are from Ref. 13 .

We first tested our PIM and AIM potentials using the polymorphs $\mathrm{B}_{2} \mathrm{O}_{3}$-I and $\mathrm{B}_{2} \mathrm{O}_{3}$-II that contain solely $\mathrm{B}_{3}$ and $\mathrm{B}_{4}$ species, respectively. The dependence of the energy $E$ on volume $V$ was determined at $0 \mathrm{~K}$ by changing isotropically the volume of the cell and optimizing the geometry of the systems. The results are shown in Fig. 3. From these plots, one can in principle extract (i) the equilibrium volume of both crystals, and (ii) the transition pressure at zero temperature (given by minus the gradient of the common tangent to the two $E(V)$ curves).$^{74}$ While it is clear that all three potentials yield similar $E(V)$ relationships for the $\mathrm{B}_{2} \mathrm{O}_{3}$-I crystal, marked differences are observed for the $\mathrm{B}_{2} \mathrm{O}_{3}$-II crystal. First, the PIMFit 1 potential is unable to stabilize the $\mathrm{B}_{2} \mathrm{O}_{3}$-II structure, i.e., an equilibrium configuration could not be obtained. This feature reflects the fact that the potential was fitted using configurations with $\mathrm{B}_{3}$ units alone, i.e., the parameters are unrepresentative of the interactions involving $\mathrm{B}_{4}$ (tetrahedral) units. In contrast, the PIM-Fit 2 potential over-stabilizes $\mathrm{B}_{2} \mathrm{O}_{3}$-II: it has a lower energy than $\mathrm{B}_{2} \mathrm{O}_{3}$-I, implying that the latter would be stable only at negative pressure. This feature reflects a tendency to over-stabilize $\mathrm{B}_{4}$ relative to $\mathrm{B}_{3}$ units. By contrast, the AIM gives the only potential for which the $E(V)$ plots are consistent with both DFT and experiments: while it underestimates the equilibrium volume of both crystals by $3 \%$, which is comparable to the accuracy of the DFT calculations, the predicted transition pressure is $1.7 \mathrm{GPa}$, in good agreement with DFT $(\sim 3 \mathrm{GPa})^{75}$ and experiments $(\sim 2 \mathrm{GPa}){ }^{76}$

We now turn to the pressure-induced $\mathrm{B}_{3}$ to $\mathrm{B}_{4}$ transition in the glass. As a first test of the quality of the potentials, the neutron structure factor $S_{\mathrm{N}}(Q)$ was computed from the MD configurations (see Ref. 13 for details), where $Q$ denotes the magnitude of the scattering vector. The results are compared to measured neutron diffraction data in Fig. 4. Data sets are reported for three different densities, which correspond experimentally to ambient pressure $\left(\rho=1.8 \mathrm{~g} \mathrm{~cm}^{-3}\right)$, a high-pressure state before the appearance of $\mathrm{B}_{4}$ units $(\rho=$ $2.4 \mathrm{~g} \mathrm{~cm}^{-3}$ ), and a high-pressure state for which half of the $\mathrm{B}_{3}$ 


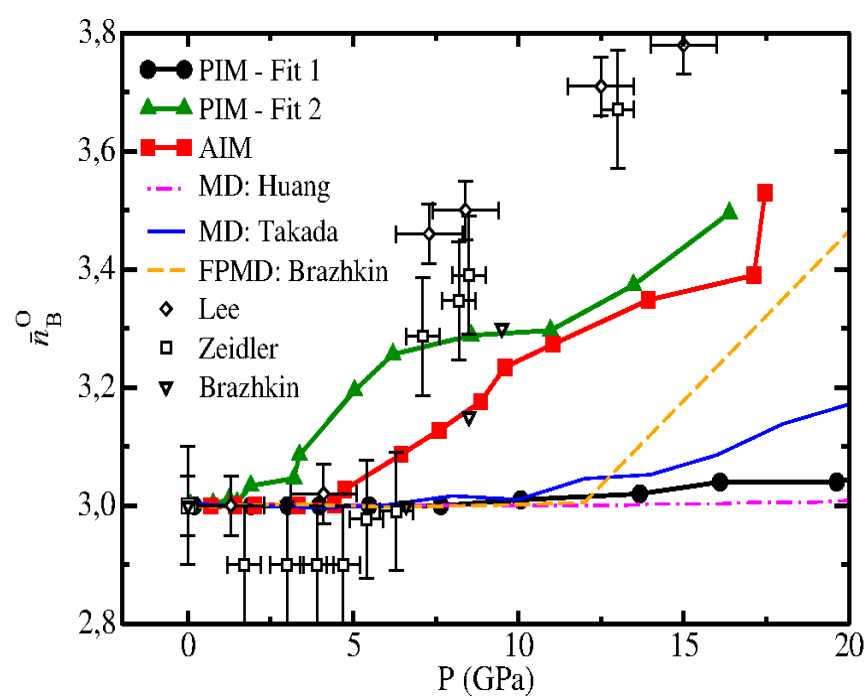

FIG. 5. Pressure dependence of the mean B-O coordination number $\bar{n}_{\mathrm{B}}^{\mathrm{O}}$ in $\mathrm{g}-\mathrm{B}_{2} \mathrm{O}_{3}$ at ambient temperature predicted by the PIMFit 1, PIM-Fit 2 and AIM potentials. The experimental results (open symbols) are from Refs. 13-15. The other MD results are from FPMD [dashed (orange) curve ${ }^{15}$ ] or from empirical force-field models [chained (purple) curve $\mathrm{e}^{39}$ and solid (blue) curve $\mathrm{e}^{73}$ ].

units have been converted into $\mathrm{B}_{4}$ tetrahedra $\left(\rho=2.7 \mathrm{~g} \mathrm{~cm}^{-3}\right)$. For each condition, the PIM-Fit 1 and AIM potentials yield structure factors in good agreement with experiment. In comparison, the PIM-Fit 2 potential leads to discrepancies with experiment that are particularly marked at the lowest density: the intensity of the first peak in $S_{\mathrm{N}}(Q)$ is too low (reflecting an incorrect reproduction of the medium-range order) and there is a small mismatch in phase at larger $Q$ values. The agreement with experiment tends to improve, however, at higher glass density.

A more stringent test of the potentials is provided by comparing the predicted pressure dependence of the boron-oxygen coordination number, $\bar{n}_{\mathrm{B}}^{\mathrm{O}}$, with the results obtained from different experimental techniques, namely neutron diffraction, ${ }^{13}$ $\mathrm{x}$-ray diffraction ${ }^{15}$ and inelastic x-ray scattering. ${ }^{14}$ As shown in Fig. 5, the potentials yield very different trends at pressures above $3 \mathrm{GPa}$. It is clear that the PIM-Fit 1 potential is unable to reproduce the $\mathrm{B}_{3}$ to $\mathrm{B}_{4}$ transition within the relevant pressure range. This failure is reminiscent of that obtained from all former classical force-fields in the literature..$^{39,73}$ In contrast, the PIM-Fit 2 and AIM potentials show unprecedented improvement, with values compatible with experiments at pressures up to $10 \mathrm{GPa}$, and falling in between the experimental and DFT-based MD results ${ }^{15}$ above $10 \mathrm{GPa}$. In the case of the PIM-Fit 2 potential, however, the threshold for the transition pressure is seemingly too low, in line with the pitfalls already evidenced in the crystalline tests, namely over-stabilization of $\mathrm{B}_{4}$ units.

Thus, it can be concluded from these first tests that PIMFit 1 provides a reasonable model for low-density conditions but fails for high-density conditions, while the situation is reversed for PIM-Fit 2 . The AIM provides the only transferable potential over the whole pressure range.

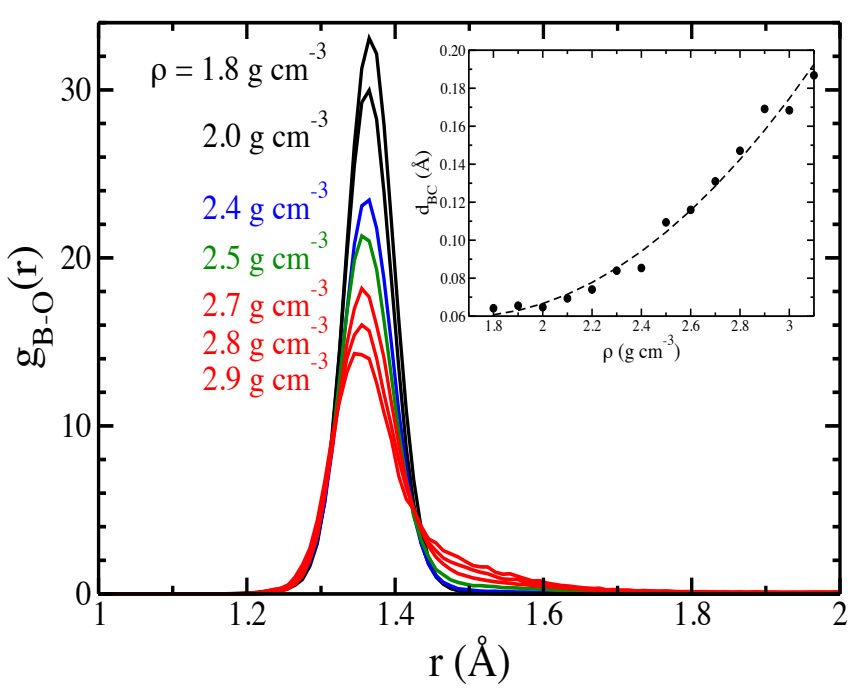

FIG. 6. Density dependence of the first peak in the B-O partial pairdistribution function $g_{\mathrm{BO}}(r)$ for $\mathrm{g}-\mathrm{B}_{2} \mathrm{O}_{3}$ obtained for the AIM potential. Inset: Density dependence of the mean distance between a boron atom $\mathrm{B}$ and center $\mathrm{C}$ of the plane formed by its three oxygen neighbors in $\mathrm{BO}_{3}$ unit.

In order to understand this observation, we analyzed in further detail the structural changes that occur during the $\mathrm{B}_{3}$ to $\mathrm{B}_{4}$ transition. Figure 6 shows the first peak of the boron-oxygen partial pair-distribution function, $g_{\mathrm{BO}}(r)$, for the AIM potential at various densities. Up to a density of $2.4 \mathrm{~g} \mathrm{~cm}^{-3}$, i.e., before the formation of $\mathrm{B}_{4}$ units, the peak shape is essentially the same (the decrease of intensity results from a renormalization effect as the density increases). At larger densities (red curves in Fig. 6), a broad shoulder appears at 1.45-1.65 A, showing the entrance of a fourth oxygen atom into the first coordination shell. This finding points to the pressure-induced formation of asymmetrical $\mathrm{B}_{4}$ units, in keeping with the observations made in neutron diffraction experiments. ${ }^{13}$ Distorted $\mathrm{B}_{4}$ units are also found in crystalline $\mathrm{B}_{2} \mathrm{O}_{3}$-II, where the B$\mathrm{O}$ bond lengths vary between 1.37 and $1.51 \AA^{3} .^{3}$ In comparison, the tetrahedral units are symmetrical in glassy silica and germania at ambient conditions, and there is only one $\mathrm{Si}-\mathrm{O}$ distance for $\alpha$-quartz (1.62 $⿱$ A) ${ }^{77}$ Another interesting effect of densification is the progressive deformation of planarity of the $\mathrm{BO}_{3}$ units. As shown first by DFT-based MD, ${ }^{17}$ and later confirmed by in situ nuclear magnetic resonance (NMR) experiments, ${ }^{78}$ the transition state between $\mathrm{B}_{3}$ and $\mathrm{B}_{4}$ is characterized by a small deviation of the boron atom from the center (C) of the plane formed by its three oxygen neighbors. The AIM captures well this behavior, as shown by the variation with density of the average B-C distance $d_{\mathrm{BC}}$ (inset to Fig. 6).

Such complex structural changes are hard to capture by simpler force fields, pointing to a possible reason for the failure of previous simulations to reproduce the $\mathrm{B}_{3}$ to $\mathrm{B}_{4}$ transition within the correct pressure range. Indeed, important ingredients of the AIM are the oxide ion breathing and deformation effects, which are included in the short-range repulsion term. In order to investigate further the impact of these effects, we calculated the pairwise repulsion forces between 


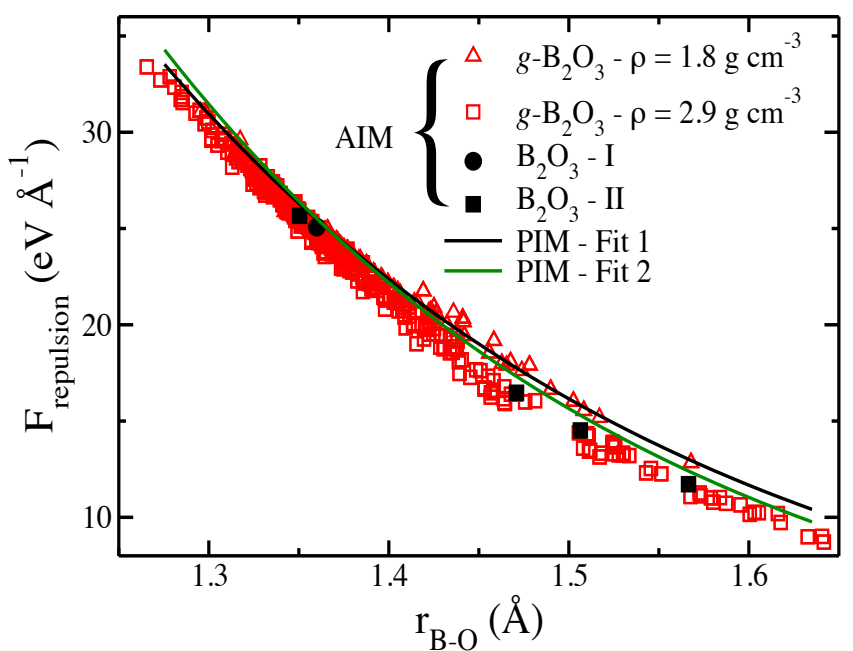

FIG. 7. Dependence of the repulsive force between all B-O firstneighbor pairs on the $\mathrm{B}-\mathrm{O}$ distance $r_{\mathrm{BO}}$. The data sets correspond to single configurations of $\mathrm{g}-\mathrm{B}_{2} \mathrm{O}_{3}$ at two different densities and to the crystalline polymorphs $\mathrm{B}_{2} \mathrm{O}_{3}$-I and $\mathrm{B}_{2} \mathrm{O}_{3}$-II. The solid curves show the BHM component $V_{\mathrm{BHM}}$ of the potentials corresponding to PIMFit 1 and PIM-Fit 2.

all nearest-neighbor B-O pairs in several configurations. Note that this is easily done despite the many-body nature of the AIM potential, because the ground-state values for all degrees of freedom were accumulated at each time step.

The results are shown Fig. 7 for two configurations of $g$ $\mathrm{B}_{2} \mathrm{O}_{3}$, with densities of $1.8 \mathrm{~g} \mathrm{~cm}^{-3}$ and $2.9 \mathrm{~g} \mathrm{~cm}^{-3}$, and for the two crystalline polymorphs. The solid lines show the BHM component $V_{\mathrm{BHM}}$ of the potentials corresponding to PIM-Fit 1 and PIM-Fit 2. We see immediately that the BHM components handle well the interaction for B-O pairs at either smaller distances (PIM-Fit 1) or at larger distances in the range 1.4-1.6 ̊̊ where the fourth oxygen ion is located (PIMFit 2), but neither of them is accurate over the entire range of distances. In the AIM, the fourth oxide ion is accommodated into the boron first-neighbor shell through a reduction in the effective radii of the oxide ions. Empirically, if the coordination polyhedra are assumed to be regular polyhedra with touching oxygen atoms, then the measured change in the $\mathrm{B}-\mathrm{O}$ bond length in $\mathrm{g}-\mathrm{B}_{2} \mathrm{O}_{3}$ from $1.35 \AA$ at ambient pressure $\left(\mathrm{B}_{3}\right)$ to $1.42 \AA$ at $17.5 \mathrm{GPa}\left(\mathrm{B}_{4}\right)^{13}$ gives a reduction of $\simeq 0.01 \AA$ in the oxygen radius. ${ }^{79}$

We note that some potentials should be able to capture these effects by fitting effectively all possible coordination environments (the family of reactive force-fields), ${ }^{80}$ although we are not aware of any attempt to model $\mathrm{B}_{2} \mathrm{O}_{3}$ within such a scheme. In the present work, the breathing and deformation effects mark the response of the ions to their changing environment, which does not need further parametrization. This feature shows that the AIM contains the correct physical ingredients for modeling $\mathrm{B}_{2} \mathrm{O}_{3}$.

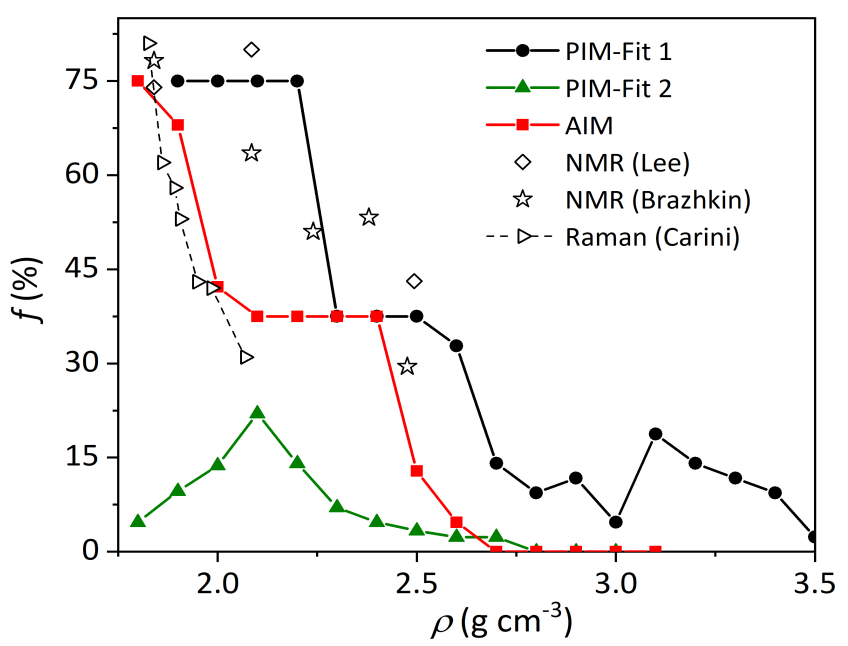

FIG. 8. The density dependence of the proportion of boron atoms in boroxol rings, $f$, in $\mathrm{g}-\mathrm{B}_{2} \mathrm{O}_{3}$. The experimental data ${ }^{12,81,82}$ correspond to ex-situ experiments, and were obtained either from quenchfrom-the-melt (under pressure) ${ }^{12,81}$ or from pressure-compacted (at a temperature below $\left.T_{\mathrm{g}}\right)^{82}$ glasses. The pressures used to compress the samples $^{12,81,82}$ were converted into densities using Fig. 1 of Ref. 13. The Raman experiments ${ }^{82}$ probe the proportion of oxygen atoms in boroxol rings, $f_{\mathrm{O}}$, which is here converted ${ }^{85}$ to the proportion of boron atoms in boroxol rings using $f=f_{\mathrm{O}} \times 1.5$.

\section{B. Boroxol rings in the glass}

As discussed in Sec. I, boroxol rings are a key feature in the structure of $\mathrm{g}-\mathrm{B}_{2} \mathrm{O}_{3}$, and their prevalence is commonly enumerated in terms of the proportion $f$ of boron atoms involved in boroxol rings. At ambient conditions, $f$ is in the range 65$80 \% .{ }^{41-43}$ Quantitative accounts of the pressure dependence of $f$ are scarce, ${ }^{12,81,82}$ but consistently show a reduction of $f$ with increasing pressure, as expected from the ring's low compacity. The pressure at which boroxol rings fully disappear $(f$ $=0$ ) is reported to be around 11-14 GPa, ${ }^{11,83}$ corresponding to a density of $2.8-3.0 \mathrm{~g} \mathrm{~cm}^{-3}$. Figure 8 compares the predictions of the models ${ }^{84}$ with the measured trend. The failure of PIM-Fit 2 is patently obvious, and accompanies its poor description of the structure factor $S_{\mathrm{N}}(Q)$ at low density (Fig. 4). The repulsive part of this potential is too strong, which impedes the stabilization of planar six-membered rings. The other two potentials provide the correct trend and are in good agreement with the available experiments, although some caution is required in making this comparison. The experimental results were obtained from ex-situ experiments for which the effects of structural relaxation are unknown. The glasses were obtained either by quenching from the melt under pressure $^{12,81}$ or by pressure-compaction at a temperature below $T_{\mathrm{g}},{ }^{82}$ and were then recovered to ambient conditions where the measurements were made.

Finally, we investigated the physical origin of the boroxol ring stability. This issue has been addressed in the past by Maranas et al. ${ }^{34}$ who pinpointed the polarization term in their potential as leading to an energy for boroxol rings that is slightly lower with respect to non-ring units. Here, we deter- 


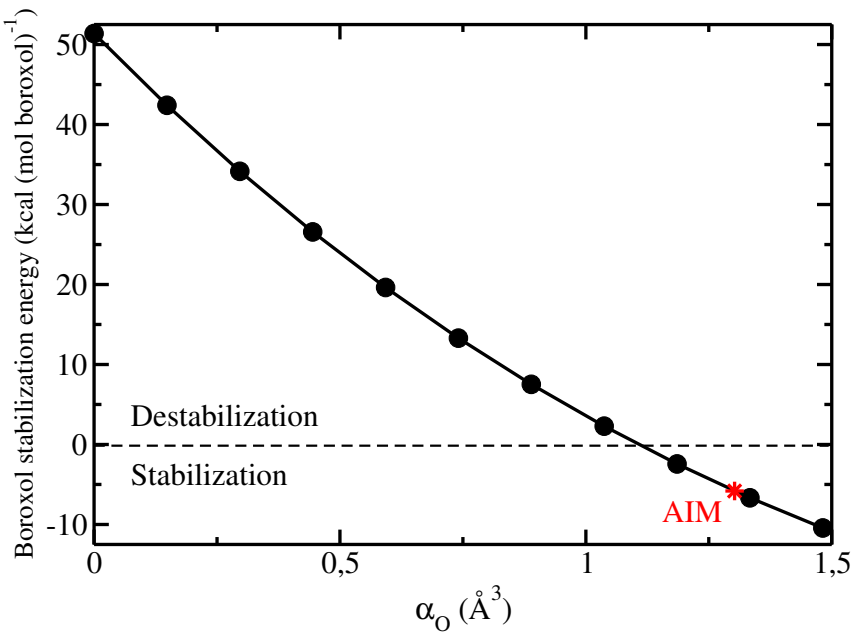

FIG. 9. Variation of the boroxol ring stabilization energy with the polarizability of the oxide ion as calculated within the framework of the AIM. The red asterisk indicates the AIM polarizability as obtained originally from fitting the DFT data.

mine the boroxol-ring stabilization energy (BSE) for a given potential in the following way. First, we computed the energy (at $0 \mathrm{~K}$ ) for a large set of glassy configurations with different proportions of boroxol rings ranging from $f=0$ to $75 \%$ (all energies were acquired at fixed density, $1.84 \mathrm{~g} \mathrm{~cm}^{-3}$ ). Second, we plotted the energies obtained as a function of $f$ and found a linear anti-correlation: the more rings in a given configuration the lower its energy (see Fig. 1 of Ref. 5 for an example). Third, we extract the BSE from the slope of this graph. We obtained BSE values of $-11.4 \mathrm{kcal}(\mathrm{mol} \text { boroxol })^{-1}$ and $-5.8 \mathrm{kcal}$ (mol boroxol) ${ }^{-1}$ for the PIM-Fit 1 and AIM potentials, respectively. ${ }^{86}$ These values encompass reasonably well the experimental estimate of $-6.4 \mathrm{kcal}$ ( $\mathrm{mol}$ boroxol) $)^{-1}$, indirectly derived from Raman measurements. ${ }^{44}$

To gain further evidence on the crucial role of polarization, we performed "numerical experiments" in which the oxide ion polarizability $\alpha_{\mathrm{O}}$ is varied, keeping all of the other parameters constant. The BSE was then determined for each of the modified force-fields. Figure 9 shows the evolution of the BSE with $\alpha_{\mathrm{O}}$ for the AIM potential. The larger the polarizability, the smaller the BSE, i.e., the greater the stability of the boroxol rings. When the polarization is turned off $\left(\alpha_{O}=0\right)$, the presence of boroxol rings increases the system energy by $51.4 \mathrm{kcal}(\mathrm{mol} \text { boroxol })^{-1}$ : running a simulation of a glass would rapidly lead to the destruction of the initial rings. As the magnitude of the polarizability is increased, ring stability sets in for $\alpha_{O} \geq 1.1 \AA^{3}$. A similar trend is observed for a similar change of $\alpha_{\mathrm{O}}$ for the PIM potentials. We recently exploited this relationship between BSE and polarizability in a study of the liquid-glass transformation: ${ }^{40}$ by artificially increasing $\alpha_{\mathrm{O}}$ in the PIM-Fit 1 potential to compensate for the limited MD equilibration time, low temperature structures with large amounts of boroxol rings were obtained.

We conclude that polarizability, an implicit many-body effect, is an essential physical ingredient in any potential aimed at reproducing the formation of boroxol rings, and thus the intermediate-range structure in $\mathrm{g}-\mathrm{B}_{2} \mathrm{O}_{3}$.

\section{CONCLUSION}

We have shown that the intriguing structural features of $\mathrm{B}_{2} \mathrm{O}_{3}$ result from a subtle balance between the various interionic interactions. In particular, the quality and transferability of the potentials that we have derived is ascribed to the inclusion of crucial many-body characteristics, namely (i) the ability for an oxide ion to "breathe" and deform, an important feature for accurately reproducing the $\mathrm{B}_{3}$ to $\mathrm{B}_{4}$ transition under pressure, and (ii) the polarizability of the oxide ion, which is at the origin of boroxol ring stability.

Our AIM potential delivers almost the same accuracy as state-of-the-art DFT but at a fraction of its computational cost. It therefore represents a significant step forward for investigating various structural problems. In particular, when used in conjunction with enhanced sampling techniques, ${ }^{87}$ it could allow for an accurate exploration of the relatively unknown $\mathrm{B}_{2} \mathrm{O}_{3}$ phase-diagram and/or the approach of the liquid to the glass transition.

\section{ACKNOWLEDGMENTS}

This work was supported by French state funds managed by the French National Research Agency (ANR) within the Investissements d'Avenir programme under reference ANR11-IDEX-0004-02, and more specifically within the framework of the Cluster of Excellence MATISSE led by Sorbonne Université. Financial support from the program PIPOG ANR-17-CE30-000 is also acknowledged. This work was performed using HPC resources from GENCITGCC/CINES/IDRIS (Grant No. A0050801875). This work was also supported by EPSRC Grant No. EP/J009741/1. AZ is supported by a Royal Society - EPSRC Dorothy Hodgkin Research Fellowship.

Appendix: Parameters used in the AIM, PIM-Fit 1 and PIM-Fit 2 potentials 
TABLE I. Parameters used in the AIM potential for $\mathrm{B}_{2} \mathrm{O}_{3}$, where all values are in atomic units.

\begin{tabular}{llllllll}
\hline \hline$A^{++}$ & 62.630 & $a^{++}$ & 3.9720 & $A^{--}$ & $2,227.6$ & $a^{--}$ & 2.6105 \\
$A^{+-}$ & 15.798 & $a^{+-}$ & 1.5465 & $B^{+-}$ & 34,636 & $b^{+-}$ & 4.8366 \\
$b_{D}^{+-}$ & 2.1152 & $b_{D}^{--}$ & 2.6858 & $c_{D}^{+-}$ & 1.2479 & $c_{D}^{--}$ \\
$C_{6}^{--}$ & 25.4 & $C_{6}^{+-}=C_{8}^{+-}$ & 0.0 & $C_{8}^{--}$ & 491.6 & $C_{6}^{++}=C_{8}^{++}$ & 0.0 \\
$b_{6}^{--}=b_{8}^{--}$ & 2.000 & $D$ & 0.6981 & $\beta$ & 1.8973 & $\zeta$ & 1.6230 \\
$\eta$ & 7.4572 & $\alpha_{\mathrm{O}}$ & 8.7893 & $q_{\mathrm{B}}$ & +3 & $q_{\mathrm{O}}$ \\
\hline \hline
\end{tabular}

TABLE II. Parameters used in the PIM-Fit 1 potential for $\mathrm{B}_{2} \mathrm{O}_{3}$, where all values are in atomic units.

\begin{tabular}{|c|c|c|c|c|c|c|c|}
\hline$\overline{A^{+-}}$ & 23.956 & $a^{+-}$ & 1.7204 & $\mathrm{~A}^{--}$ & 464.06 & $a^{--}$ & 2.6576 \\
\hline$A^{++}$ & 1,015 & $a^{++}$ & 6.467 & & & & \\
\hline$C_{6}^{--}$ & 25.4 & $C_{6}^{+-}=C_{8}^{+-}$ & 0.0 & $C_{8}^{--}$ & 491.6 & $C_{6}^{++}=C_{8}^{++}$ & 0.0 \\
\hline
\end{tabular}

TABLE III. Parameters used in the PIM-Fit 2 potential for $\mathrm{B}_{2} \mathrm{O}_{3}$, where all values are in atomic units. Cations interact solely via their strongly repulsive Coulomb interaction.

\begin{tabular}{llllllll}
\hline \hline$A^{+-}$ & 31.209 & $a^{+-}$ & 1.8466 & $A^{--}$ & 201.66 & $a^{--}$ & 2.2121 \\
$b_{D}^{+-}$ & 2.0564 & $b_{D}^{--}$ & 2.975 & $c_{D}^{+-}$ & 1.2172 & $c_{D}^{--}$ & 3.3595 \\
$C_{6}^{--}$ & 22.186 & $C_{6}^{+-}=C_{8}^{+-}$ & 0.0 & $C_{8}^{--}$ & 426.65 & $C_{6}^{++}=C_{8}^{++}$ & 0.0 \\
$b_{6}^{--}=b_{8}^{--}$ & 1.400 & $\alpha_{\mathrm{O}}$ & 9.868 & $q_{\mathrm{B}}$ & +3 & $q_{\mathrm{O}}$ & -2 \\
\hline \hline
\end{tabular}


${ }^{1}$ A. C. Wright, Phys. Chem. Glasses: Eur. J. Glass Sci. Technol. B 59, 65 (2018).

${ }^{2}$ G. E. Gurr, P. W. Montgomery, C. D. Knutson, and B. T. Gorres, Acta Crystallogr. B 26, 906 (1970).

${ }^{3}$ C. T. Prewitt and R. D. Shannon, Acta Crystallogr. B 24, 869 (1968).

${ }^{4}$ D. R. Uhlmann, J. F. Hays, and D. Turnbull, Phys. Chem. Glasses 8, 1 (1967).

${ }^{5}$ G. Ferlat, T. Charpentier, A. P. Seitsonen, A. Takada, M. Lazzeri, L. Cormier, G. Calas, and F. Mauri, Phys. Rev. Lett. 101, 065504 (2008).

${ }^{6}$ A. Takada, C. R. A. Catlow, and G. D. Price, J. Phys.: Condens. Matter 7, 8659 (1995)

${ }^{7}$ A. Takada, C. R. A. Catlow, and G. D. Price, Phys. Chem. Glasses 44, 147 (2003).

${ }^{8}$ F. Claeyssens, N. L. Allan, N. C. Norman, and C. A. Russell, Phys. Rev. B 82, 094119 (2010).

${ }^{9}$ G. Ferlat, A. P. Seitsonen, M. Lazzeri, and F. Mauri, Nature Mat. 11, 925 (2012).

${ }^{10} \mathrm{G}$. Ferlat, in Molecular Dynamics Simulations of Disordered Materials: From Network Glasses to Phase-Change Memory Alloys, Springer Series in Materials Science, Vol. 215, edited by Massobrio, C. and Du, J. and Bernasconi, M. and Salmon, P. S. (Springer, Cham, Switzerland, 2015) Chap. 14, pp. 367-414.

${ }^{11}$ J. Nicholas, S. Sinogeikin, J. Kieffer, and J. Bass, Phys. Rev. Lett. 92, 215701 (2004).

${ }^{12}$ S. K. Lee, K. Mibe, Y. Fei, G. D. Cody, and B. O. Mysen, Phys. Rev. Lett. 94, 165507 (2005).

${ }^{13}$ A. Zeidler, K. Wezka, D. A. J. Whittaker, P. S. Salmon, A. Baroni, S. Klotz, H. E. Fischer, M. C. Wilding, C. L. Bull, M. G. Tucker, M. Salanne, G. Ferlat, and M. Micoulaut, Phys. Rev. B 90, 024206 (2014).

${ }^{14}$ S. K. Lee, P. J. Eng, H.-K. Mao, Y. Meng, M. Newville, M. H. Hu, and J. Shu, Nature Mat. 4, 851 (2005).

${ }^{15}$ V. V. Brazhkin, Y. Katayama, K. Trachenko, O. B. Tsiok, A. G. Lyapin, E. Artacho, M. Dove, G. Ferlat, Y. Inamura, and H. Saitoh, Phys. Rev. Lett. 101, 035702 (2008).

${ }^{16}$ P. Umari and A. Pasquarello, Phys. Rev. Lett. 95, 137401 (2005).

${ }^{17}$ K. Trachenko, V. V. Brazhkin, G. Ferlat, M. T. Dove, and E. Artacho, Phys. Rev. B 78, 172102 (2008).

${ }^{18}$ S. Ohmura and F. Shimojo, Phys. Rev. B. 78, 224206 (2008); 80, 020202 (2009); 81, 014208 (2010).

${ }^{19}$ C. Scherer, F. Schmid, M. Letz, and J. Horbach, Comput. Mater. Sci. 159, 73 (2019).

${ }^{20}$ T. F. Soules, J. Chem. Phys. 73, 4032 (1980).

${ }^{21}$ M. Amini, S. K. Mitra, and R. W. Hockney, J. Phys. C: Solid State Phys. 14, 3689 (1981).

${ }^{22}$ Q. Xu, K. Kawamura, and T. Yokokawa, J. Non-Cryst. Solids 104, 261 (1988).

${ }^{23}$ W. Soppe, C. Van Der Marel, and H. W. den Hartog, J. Non-Cryst. Solids 101, 101 (1988); W. Soppe and H. W. den Hartog, 108, 260 (1989); W. Soppe, C. Van Der Marel, W. F. van Gunsteren, and H. W. den Hartog, 103, 201 (1988).

${ }^{24}$ H. Inoue, N. Aoki, and I. Yasui, J. Am. Ceram. Soc. 70, 622 (1987).

${ }^{25}$ A. H. Verhoef and H. W. den Hartog, Radiation Effects and Defects in Solids 119, 493 (1991); J. Non-Cryst. Solids 146, 267 (1992); 180, 102 (1994).

${ }^{26}$ A. Takada, C. R. A. Catlow, and G. D. Price, J. Phys.: Condens. Matter 7, 8693 (1995)

${ }^{27}$ R. Fernández-Perea, F. J. Bermejo, and E. Enciso, Phys. Rev. B 53, 6215 (1996).

${ }^{28}$ F. J. Bermejo, J. Dawidowski, R. Fernández-Perea, and J. L. Martínez, Phys. Rev. B 54, 244 (1996).

${ }^{29}$ R. E. Youngman, J. Kieffer, J. D. Bass, and L. Duffrène, J. Non-Cryst. Solids 222, 190 (1997).

${ }^{30}$ R. Fernández-Perea, F. J. Bermejo, and M. L. Senent, Phys. Rev. B 54, 6039 (1996).

${ }^{31}$ E. Kashchieva, B. Shivachev, and Y. Dimitriev, J. Non-Cryst. Solids 351, 1158 (2005).

${ }^{32}$ A. Takada, Phys. Chem. Glasses: Eur. J. Glass Sci. Technol. B 47, 493 (2006).

${ }^{33} \mathrm{M}$. Teter, in Borate Glasses, Crystals and Melts, edited by A. C. Wright, S. A. Feller, and A. C. Hannon (Soc. Glass Tech., Sheffield, 1997) p. 407.
${ }^{34}$ J. K. Maranas, Y. Chen, D. K. Stillinger, and F. H. Stillinger, J. Chem. Phys. 115, 6578 (2001).

${ }^{35}$ S. K. Fullerton and J. K. Maranas, J. Chem. Phys. 121, 8562 (2004).

${ }^{36}$ S. K. Fullerton and J. K. Maranas, Nano. Lett. 5, 363 (2005).

${ }^{37}$ L. Huang and J. Kieffer, Phys. Rev. B 74, 224107 (2006).

${ }^{38}$ L. Huang, M. Durandurdu, and J. Kieffer, J. Phys. Chem. C 111, 13712 (2007).

${ }^{39}$ L. Huang, J. Nicholas, J. Kieffer, and J. Bass, J. Phys. : Condens. Matter 20, 075107 (2008).

${ }^{40}$ O. L. G. Alderman, G. Ferlat, A. Baroni, M. Salanne, M. Micoulaut, C. J. Benmore, A. Lin, A. Tamalonis, and J. K. R. Weber, J. Phys. Condens. Matter 27, 455104 (2015).

${ }^{41}$ A. C. Hannon, D. I. Grimley, R. A. Hulme, A. C. Wright, and R. N. Sinclair, J. Non-Cryst. Solids 177, 299 (1994).

${ }^{42}$ R. E. Youngman, S. T. Haubrich, J. W. Zwanziger, M. T. Janicke, and B. F. Chmelka, Science 269, 1416 (1995).

${ }^{43}$ C. Joo, U. Werner-Zwanziger, and J. W. Zwanziger, J. Non-Cryst. Solids 271, 265 (2000).

${ }^{44}$ G. E. Walrafen, S. R. Samanta, and P. N. Krishnan, J. Chem. Phys. 72, 113 (1980).

${ }^{45}$ G. E. Walrafen, M. S. Hokmabadi, P. N. Krishnan, S. Guha, and R. G. Munro, J. Chem. Phys. 79, 3609 (1983).

${ }^{46}$ A. K. Soper, J. Phys.: Condens. Matter 22, 404210 (2010).

${ }^{47}$ F. Pacaud, J.-M. Delaye, T. Charpentier, L. Cormier, and M. Salanne, J. Chem. Phys. 147, 161711 (2017).

${ }^{48}$ M. Born and J. E. Mayer, Z. Phys. A - Hadrons Nucl. 75, 1 (1932).

${ }^{49}$ J. E. Mayer, J. Chem. Phys. 1, 270 (1933).

${ }^{50}$ M. L. Huggins and J. E. Mayer, J. Chem. Phys. 1, 643 (1933).

${ }^{51}$ B. W. H. van Beest, G. J. Kramer, and R. A. van Santen, Phys. Rev. Lett. 64, 1955 (1990).

${ }^{52}$ A. Carré, J. Horbach, S. Ispas, and W. Kob, Europhys. Lett. 82, 17001 (2008).

${ }^{53}$ F. Pacaud and M. Micoulaut, J. Chem. Phys. 143, 064502 (2015).

${ }^{54}$ K. T. Tang and J. P. Toennies, J. Chem. Phys. 80, 3726 (1984).

${ }^{55}$ P. A. Madden and M. Wilson, Chem. Soc. Rev. 25, 339 (1996).

${ }^{56}$ M. Wilson, P. A. Madden, M. Hemmati, and C. A. Angell, Phys. Rev. Lett. 77, 4023 (1996).

${ }^{57}$ M. Wilson and P. A. Madden, Faraday Discuss. 106, 339 (1997).

${ }^{58}$ A. J. Rowley, P. Jemmer, M. Wilson, and P. A. Madden, J. Chem. Phys. 108, 10209 (1998).

${ }^{59}$ A. Aguado and P. A. Madden, Phys. Rev. B 70, 245103 (2004).

${ }^{60}$ S. Jahn, P. A. Madden, and M. Wilson, Phys. Rev. B 74, 024112 (2006).

${ }^{61}$ S. Jahn and P. A. Madden, Phys. Earth Planet. Inter. 162, 129 (2007).

${ }^{62}$ P. Cieplak, F. Y. Dupradeau, Y. Duan, and J. Wang, J. Phys. Condens. Matter 21, 333102 (2009).

${ }^{63}$ A. Aguado, L. Bernasconi, S. Jahn, and P. A. Madden, Faraday Discuss. 124, 171 (2003).

${ }^{64}$ B. Rotenberg, M. Salanne, C. Simon, and R. Vuilleumier, Phys. Rev. Lett. 104, 138301 (2010).

${ }^{65}$ M. Salanne, D. Marrocchelli, and G. W. Watson, J. Phys. Chem. C 116, 18618 (2012).

${ }^{66}$ J. P. Perdew, K. Burke, and M. Ernzerhof, Phys. Rev. Lett. 77, 3865 (1996).

${ }^{67}$ The glassy configurations used for the parameter calibration were obtained from the quench-from-the melt technique. In consequence, the proportion of boroxol rings $(f=22 \%)$ in these configurations does not match the expected experimental value $(f \simeq 75 \%)$. However, the model contained threefold coordinated boron atoms in both boroxol ring and non-ring environments, which are therefore accounted for in the parameter calibration.

${ }^{68}$ P. L. Silvestrelli, Phys. Rev. Lett. 100, 053002 (2008).

${ }^{69}$ H. Hay, G. Ferlat, M. Casula, A. P. Seitsonen, and F. Mauri, Phys. Rev. B 92, 144111 (2015).

${ }^{70}$ G. Ferlat, M. Hellgren, F.-X. Coudert, H. Hay, F. Mauri, and M. Casula, Phys. Rev. Materials 3, 063603 (2019).

${ }^{71}$ T. Laino and J. Hutter, J. Chem. Phys. 129, 074102 (2008).

${ }^{72}$ L.-H. Kieu, J.-M. Delaye, L. Cormier, and C. Stolz, J. Non-Cryst. Solids 357, 3313 (2011).

${ }^{73}$ A. Takada, Phys. Chem. Glasses 45, 156 (2004).

${ }^{74}$ A. Mujica, A. Rubio, A. Muñoz, and R. J. Needs, Rev. Modern Phys. 75, 863 (2003).

${ }^{75}$ U. Engberg, Phys. Rev. B 55, 2824 (1997). 
${ }^{76}$ J. D. Mackenzie and W. F. Claussen, J. Am. Ceram. Soc. 44, 79 (1961).

${ }^{77}$ H. D'Amour, W. Denner, and H. Schulz, Acta Crystallogr. B 35, 550 (1979).

${ }^{78}$ T. Edwards, T. Endo, J. H. Walton, and S. Sen, Science 345, 1027 (2014).

${ }^{79}$ A. Zeidler, P. S. Salmon, and L. B. Skinner, Proc. Natl. Acad. Sci. USA 111, 10045 (2014).

${ }^{80}$ A. C. T. van Duin, S. Dasgupta, F. Lorant, and W. A. Goddard III, J. Phys. Chem. A 105, 9396 (2001).

${ }^{81}$ V. V. Brazhkin, I. Farnan, K.-I. Funakoshi, M. Kanzaki, Y. Katayama, A. G. Lyapin, and H. Saitoh, Phys. Rev. Lett. 105, 115701 (2010).

${ }^{82}$ G. Carini Jr, G. Carini, G. D’Angelo, M. Federico, and V. Romano, J. Non-Cryst. Solids 492, 102 (2018).

${ }^{83}$ M. Grimsditch, A. Polian, and A. C. Wright, Phys. Rev. B. 54, 152 (1996).

${ }^{84}$ In the calculated structures, all $\mathrm{B}_{3} \mathrm{O}_{3}$ rings were counted as boroxol rings, irrespective of their planarity.
${ }^{85}$ For boroxol rings $\left(\mathrm{B}_{3} \mathrm{O}_{3}\right), N_{\mathrm{O} \text {,boroxol }}=N_{\mathrm{B}, \text { boroxol }}$, where $N_{\mathrm{O} \text {,boroxol }}$ and $N_{\mathrm{B} \text {,boroxol }}$ are the numbers of oxygen and boron atoms in boroxol rings, respectively. For g- $\mathrm{B}_{2} \mathrm{O}_{3}, N_{\mathrm{O}}=1.5 N_{\mathrm{B}}$ where $N_{\mathrm{O}}$ and $N_{\mathrm{B}}$ are the total numbers of boron and oxygen atoms in the glass, respectively. It follows that the proportion of boron atoms in boroxol rings $f=N_{\mathrm{B}, \text { boroxol }} / N_{\mathrm{B}}=f_{\mathrm{O}} N_{\mathrm{O}} / N_{\mathrm{B}}=$ $f_{\mathrm{O}} \times 1.5$.

${ }^{86}$ The slopes obtained with the PIM-Fit 1 and AIM potentials are $-5.7 \mathrm{kcal}\left(\mathrm{mol} \mathrm{B}_{2} \mathrm{O}_{3}\right)^{-1}$ and $-2.9 \mathrm{kcal}\left(\mathrm{mol} \mathrm{B}_{2} \mathrm{O}_{3}\right)^{-1}$, respectively. These values convert to $-5.7 / 0.75=-7.6 \mathrm{kcal}\left(\mathrm{mol} \mathrm{B}_{2} \mathrm{O}_{3} \text { in boroxol) }\right)^{-1}$ and $-2.9 / 0.75=-3.9 \mathrm{kcal}\left(\mathrm{mol} \mathrm{B}_{2} \mathrm{O}_{3} \text { in boroxol }\right)^{-1}$, respectively, if the glass has $75 \%$ of boron atoms in boroxol rings. Further, since one mole of boroxol units $\left(\mathrm{B}_{3} \mathrm{O}_{9 / 2}\right)$ is equivalent to 1.5 moles of $\mathrm{B}_{2} \mathrm{O}_{3}$, one obtains BSE values of $-11.4 \mathrm{kcal}\left(\mathrm{mol}\right.$ boroxol) ${ }^{-1}$ for PIM-Fit 1 and $-5.8 \mathrm{kcal}$ (mol boroxol) ${ }^{-1}$ for AIM.

${ }^{87}$ S. Pipolo, M. Salanne, G. Ferlat, S. Klotz, A. M. Saitta, and F. Pietrucci, Phys. Rev. Lett. 119, 245701 (2017). 